\title{
High teenage BMI increases ESRD risk
}

Obesity in adolescence is associated with an increased risk of end-stage renal disease (ESRD) in later life, show the results of a new cohort study in the Israeli population. This association was observed both in the presence and in the absence of diabetes mellitus.

"The association between adolescent BMI and future risk of ESRD is not fully established or understood," comments study researcher Asaf Vivante. "The contribution of diabetes-related ESRD to such a possible association is also uncertain," he adds.

Vivante et al. analysed medical data of $1,194,704$ male and female individuals aged 17 years who underwent health checks in 1967-1997 to assess their suitability for military service. The mean follow-up period was 25.51 person-years, during which 713 male and 161 female individuals (total 874) developed ESRD, as recorded in the Israeli ESRD registry. Overweight and obesity were defined as
$\mathrm{BMI} \geq 85^{\text {th }}$ and $<95^{\text {th }}$ age-specific and sexspecific percentiles, and $\geq 95^{\text {th }}$ age-specific and sex-specific percentile, respectively.

The risk of future ESRD was increased among adolescents with overweight (HR 3.00) and obesity (HR 6.89). Excessive weight was associated with increased risk of both diabetes-related ESRD and diabetes-unrelated ESRD. "Demonstrating the association of ESRD with elevated BMI in adolescence supports the notion that overweightrelated kidney damage may already begin during childhood," says Vivante. "A number of processes in the pathway leading to nephron loss might be accelerated with increased BMI, even in the absence of diabetic nephropathy."

Joana Osório

Original article Vivante, A. et al. Body mass index in

1.2 million adolescents and risk for end-stage renal disease.

Arch. Intern. Med. doi:10.1001/2013.jamainternmed.85 